\title{
Educational Assessment Is an Enduring Theme of Numeracy
}

\section{H. L. Vacher}

University of South Florida, vacher@usf.edu

Follow this and additional works at: https://digitalcommons.usf.edu/numeracy

Part of the Educational Assessment, Evaluation, and Research Commons

\section{Recommended Citation}

Vacher, H. L.. "Educational Assessment Is an Enduring Theme of Numeracy." Numeracy 8, Iss. 1 (2015): Article 1. DOI: http://dx.doi.org/10.5038/1936-4660.8.1.1 


\title{
Educational Assessment Is an Enduring Theme of Numeracy
}

\begin{abstract}
The Assessment Theme Collection in this issue brings the count of papers on QL assessment to 22 out of the 136 papers (16.2\%) in the journal's first 15 issues. After the first ten issues (our first five years), the counts were 13 and 85 respectively (15.1\%). A table in this editorial updates the list of our papers on the subject.
\end{abstract}

Keywords

assessment, quantitative literacy

Creative Commons License

(c) (i) (9)

This work is licensed under a Creative Commons Attribution-Noncommercial 4.0 License

\section{Cover Page Footnote}

Len Vacher is a geology professor in the School of Geosciences at the University of South Florida and is co-editor of this journal. 
This issue includes an Assessment Theme Collection containing five papers on QL-educational assessment. This collection comes to us largely thanks to Associate Editor Donna Sundre who solicited the articles and managed them through the rigorous process of peer-review and revision. Two more papers are in the final stages of completion, and we look forward to including them in the July issue of Numeracy.

Educational assessment has been an enduring theme in Numeracy. The editorial "Scope of Numeracy after Five Years" (v. 6, issue 1, Jan 2013) identified educational assessment as one of five subject areas that clearly emerged as themes in the first ten issues of the journal. Overall, there were 85 papers (editorials, articles, perspectives, book reviews, and columns) in those issues, and 13 (15.3\%) of them were identified in the editorial as educational-assessment papers. The other popular subjects were QL and writing (12 papers), the QL construct (20), focused QL courses and curricula (12), and QL across the curriculum (12).

Since then we have published three more educational-assessment papers amongst the 37 papers of 2013 and 2014, and with this issue we are publishing six more (counting this editorial) amongst 14 papers. Thus with this $15^{\text {th }}$ issue, Numeracy has published 136 papers, and $22(16.2 \%)$ of them are on educational assessment. They are listed in the appendix.

At the end of 2014, the count of downloads for Numeracy papers was 87,681. Of these, 13,794 (15.7\%) were for the 16 educational-assessment articles.

\section{Appendix}

\section{$\underline{\text { Educational assessment }}$}

\begin{tabular}{|c|c|c|c|}
\hline $\begin{array}{l}\text { Vacher and } \\
\text { Chavez }\end{array}$ & 2009 & $\begin{array}{l}\text { Quantitative literacy on the Web of Science, } 2 \text { - } \\
\text { Mining the health numeracy literature for } \\
\text { assessment items }\end{array}$ & $\underline{\text { http://dx.doi.org/10.5038/1936- }}$ \\
\hline Taylor & 2009 & Assessing quantitative reasoning (Editorial) & $\underline{\text { http://dx.doi.org/10.5038/1936- }}$ \\
\hline $\begin{array}{l}\text { Wallace et } \\
\text { al. }\end{array}$ & 2009 & $\begin{array}{l}\text { Quantitative literacy assessments: An } \\
\text { introduction to testing tests }\end{array}$ & $\underline{\text { http://dx.doi.org/10.5038/1936- }}$ \\
\hline Grawe et al. & 2010 & $\begin{array}{l}\text { A rubric for assessing quantitative reasoning in } \\
\text { written arguments }\end{array}$ & $\underline{\text { http://dx.doi.org/10.5038/1936- }}$ \\
\hline $\begin{array}{l}\text { Sundre and } \\
\text { Thelk }\end{array}$ & 2010 & $\begin{array}{l}\text { Advancing Assessment of Quantitative and } \\
\text { Scientific Reasoning }\end{array}$ & $\frac{\text { http://dx.doi.org/10.5038/1936- }}{\underline{4660.3 .2 .2}}$ \\
\hline $\begin{array}{l}\text { Steele and } \\
\text { Kilic-Bahi }\end{array}$ & 2010 & $\begin{array}{l}\text { Quantitative literacy: Does it work? Evaluation of } \\
\text { student outcomes at Colby-Sawyer College }\end{array}$ & $\underline{\text { http://dx.doi.org/10.5038/1936- }}$ \\
\hline Wetzel & 2011 & $\begin{array}{l}\text { Spreadsheets Across the Curriculum, 2: } \\
\text { Assessing our success with students at Eckerd } \\
\text { College }\end{array}$ & $\underline{\text { http://dx.doi.org/10.5038/1936- }}$ \\
\hline
\end{tabular}




\begin{tabular}{|c|c|c|c|}
\hline $\begin{array}{l}\text { Kosko and } \\
\text { Wilkins }\end{array}$ & 2011 & $\begin{array}{l}\text { Communicating quantitative literacy: An } \\
\text { examination of open-ended assessment items in } \\
\text { TIMSS, NALS, IALS, and PISA }\end{array}$ & $\begin{array}{l}\text { http://dx.doi.org/10.5038/1936- } \\
\underline{4660.4 .2 .3}\end{array}$ \\
\hline Ward et al. & 2011 & $\begin{array}{l}\text { Development of an assessment of quantitative } \\
\text { literacy for Miami University }\end{array}$ & $\underline{\text { http://dx.doi.org/10.5038/1936- }}$ \\
\hline $\begin{array}{l}\text { Sikorskii et } \\
\text { al. }\end{array}$ & 2011 & $\begin{array}{l}\text { Quantitative literacy at Michigan State } \\
\text { University, 1: Development and initial evaluation } \\
\text { of the assessment }\end{array}$ & $\begin{array}{l}\text { http://dx.doi.org/10.5038/1936- } \\
\underline{4660.4 .2 .5}\end{array}$ \\
\hline Hassad & 2011 & $\begin{array}{l}\text { Constructivist and behaviorist approaches: } \\
\text { Development and initial evaluation of a teaching } \\
\text { practice scale for introductory statistics at the } \\
\text { college level }\end{array}$ & $\begin{array}{l}\text { http://dx.doi.org/10.5038/1936- } \\
\text { 4660.4.2.7 }\end{array}$ \\
\hline $\begin{array}{l}\text { Boersma et } \\
\text { al. }\end{array}$ & 2011 & $\begin{array}{l}\text { Quantitative Reasoning in the Contemporary } \\
\text { World, 3: Assessing student learning }\end{array}$ & $\begin{array}{l}\text { http://dx.doi.org/10.5038/1936- } \\
\underline{4660.4 .2 .8}\end{array}$ \\
\hline $\begin{array}{l}\text { Lehto and } \\
\text { Vacher }\end{array}$ & 2012 & $\begin{array}{l}\text { Spreadsheets Across the Curriculum, } 4 \text { : Evidence } \\
\text { of student learning and attitudes about } \\
\text { spreadsheets in a physical geology course }\end{array}$ & $\begin{array}{l}\text { http://dx.doi.org/10.5038/1936- } \\
\underline{4660.5 .2 .5}\end{array}$ \\
\hline $\begin{array}{l}\text { Boersma } \\
\text { and Klyve }\end{array}$ & 2013 & $\begin{array}{l}\text { Measuring habits of mind: Toward a prompt-less } \\
\text { instrument for assessing quantitative literacy }\end{array}$ & $\begin{array}{l}\text { http://dx.doi.org/10.5038/1936- } \\
\underline{4660.6 .1 .6}\end{array}$ \\
\hline $\begin{array}{l}\text { Grawe and } \\
\text { Grawe }\end{array}$ & 2014 & $\begin{array}{l}\text { Reflections on the introduction of quantitative } \\
\text { assessment in persuasive writing classes }\end{array}$ & $\underline{\text { http://dx.doi.org/10.5038/1936- }}$ \\
\hline Gaze et al. & 2014 & $\begin{array}{l}\text { Towards developing a Quantitative } \\
\text { Literacy/Reasoning Assessment instrument }\end{array}$ & $\begin{array}{l}\text { http://dx.doi.org/10.5038/1936- } \\
\text { 4660.7.2.4 }\end{array}$ \\
\hline Vacher & 2015 & $\begin{array}{l}\text { Educational assessment is an enduring theme of } \\
\text { Numeracy (Editorial) }\end{array}$ & $\begin{array}{l}\text { http://dx.doi.org/10.5038/1936- } \\
\underline{4660.8 .1 .1}\end{array}$ \\
\hline $\begin{array}{l}\text { Hathcoat et } \\
\text { al. }\end{array}$ & 2015 & $\begin{array}{l}\text { Assessing college students' quantitative and } \\
\text { scientific reasoning: The James Madison } \\
\text { University story }\end{array}$ & $\underline{\text { http://dx.doi.org/10.5038/1936- }}$ \\
\hline Gittens & 2015 & $\begin{array}{l}\text { Assessing numeracy in the upper elementary and } \\
\text { middle school years }\end{array}$ & $\underline{\text { http://dx.doi.org/10.5038/1936- }}$ \\
\hline $\begin{array}{l}\text { Gillmor et } \\
\text { al. }\end{array}$ & 2015 & $\begin{array}{l}\text { Effects of reducing the cognitive load of } \\
\text { mathematics test items on student performance }\end{array}$ & $\underline{\text { http://dx.doi.org/10.5038/1936- }}$ \\
\hline $\begin{array}{l}\text { Dumford } \\
\text { and Rocconi }\end{array}$ & 2015 & $\begin{array}{l}\text { Development of the quantitative reasoning items } \\
\text { on the National Survey of Student Engagement }\end{array}$ & $\underline{\text { http://dx.doi.org/10.5038/1936- }}$ \\
\hline $\begin{array}{l}\text { Wright and } \\
\text { Howard }\end{array}$ & 2015 & $\begin{array}{l}\text { Assessment of improvement: Two models for } \\
\text { assessing a large quantitative reasoning } \\
\text { requirement }\end{array}$ & $\underline{\text { http://dx.doi.org/10.5038/1936- }}$ \\
\hline
\end{tabular}

